EPJ Web of Conferences 41, 08007 (2013)

DOI: $10.1051 /$ epjconf/20134108007

(C) Owned by the authors, published by EDP Sciences, 2013

\title{
Dark excited states of carotenoid in light harvesting complex probing with femtosecond stimulated Raman spectroscopy
}

\author{
O. Yoshimatsu ${ }^{1}$, K. Abe ${ }^{1,2}$, S. Sakai ${ }^{3}$, T. Horibe ${ }^{2,4}$, R. Fujii ${ }^{2,5}$, M. Nango ${ }^{2,5}$, H. Hashimoto ${ }^{2,4,5}$, and \\ M. Yoshizawa ${ }^{1,2}$ \\ ${ }^{1}$ Dep. of Physics, Graduate School of Science, Tohoku University, Japan \\ ${ }^{2}$ JST, CREST, Japan \\ ${ }^{3}$ Dep. of Life and Materials Engineering, Nagoya Institute of Technology, Japan. \\ ${ }^{4}$ Dep. of Physics, Graduate School of Science, Osaka City University, Japan. \\ ${ }^{5}$ OCARINA, Osaka City University, Japan.
}

\begin{abstract}
Vibrational dynamics of dark excited states in carotenoids have been investigated using tunable Raman pump pulses. The $\mathrm{S}_{1}$ state has same vibrational dynamics in light-harvesting complex (LH1) and solution. The $\mathrm{S}^{*}$ state in LH1 has similar vibrational modes with the triplet state of carotenoid. However, the so-called $\mathrm{S}^{*}$ state in solution does not have the modes and is concluded to be different from the $\mathrm{S}^{*}$ state in LH1.
\end{abstract}

\section{Introduction}

In light harvesting (LH) complexes of photosynthetic purple bacteria, light energy is absorbed by carotenoid (Car) and transferred to nearby bacteriochlorophyll (BChl) as shown in Fig.1. After photoexcitation to the optically allowed $S_{2}$ state of carotenoid, internal conversion to the optically forbidden $S_{1}$ state takes place within a few hundred femtoseconds. The following internal conversion to the $\mathrm{S}_{0}$ ground state occurs on a picoseconds timescale. Ultrafast spectroscopic studies have shown that the excitation energy transfer to BChl takes place from $\mathrm{S}_{2}$ and/or $\mathrm{S}_{1}$ competing with these internal conversion processes $[1,2]$. Besides these singlet states, it has been reported that an additional optically forbidden state, which is termed $\mathrm{S}^{*}$, have considerable importance in the light-harvesting function [3,4]. It is the precursor on the reaction pathway toward triplet formation and plays a critical role in efficient excitation energy deactivation in the LH1 complex [5]. However, the entire picture of ultrafast
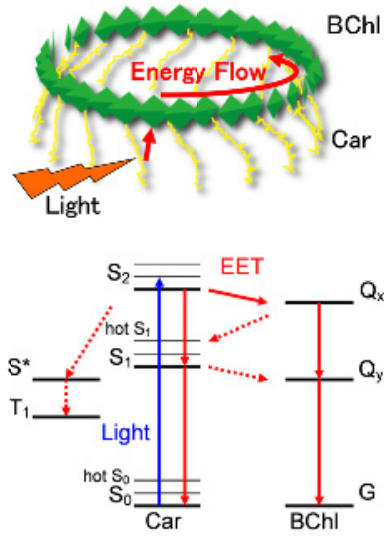

Fig. 1. Schematic picture and energy level diagram of LH1. energy flow in the LH complex has not been obtained yet.

In this study, vibrational dynamics of spirilloxanthin in LH1 complex and cyclohexane solution have been investigated by femtosecond stimulated Raman spectroscopy (FSRS) [6]. The dlark excited states $\left(\mathrm{S}_{1}\right.$ and $\left.\mathrm{S}^{*}\right)$ are selectively observed using tunable Raman pump pulses resonant to the transient absorption.

This is an Open Access article distributed under the terms of the Creative Commons Attribution License 2.0, which permits unrestricted use, distribution, and reproduction in any medium, provided the original work is properly cited. 


\section{Experimental}

The native LH1 complex from Rhodospirillum rubrum S1 contains spirilloxanthin as a major carotenoid. In this study, the reconstituted LH1 with purified spirilloxanthin was prepared as described elsewhere [7] to investigate the effect due to other kinds of carotenoids that may be involved. The solution of LH1(Spx) was dispersed in a poly-vinyl alcohol film on a glass plate. The solution of the native LH1 was measured using a $1 \mathrm{~mm}$ optical path length cell. During the laser spectroscopic measurements, the sample was translated to avoid sample degradation and the accumulation of any potential photoproducts.

The femtosecond absorption and stimulated Raman spectroscopy setup was based on an amplified mode-locked Ti:Sapphire laser system. Two independent optical parametric amplifiers were used to generate the first pump and Raman pump pulses. The first pump pulse was resonant to $S_{2}$ of spirilloxanthin (500 and $540 \mathrm{~nm}, 100 \mathrm{fs}$ ) and the tunable narrowband Raman pump pulse (560-620 $\mathrm{nm}, 20 \mathrm{~cm}^{-1}$ ) was resonant to $\mathrm{S}_{1}$ or $\mathrm{S}^{*}$ of spirilloxanthin. A probe pulse after the sample was collected at $1 \mathrm{kHz}$ repetition rate. Noise level of the obtained absorbance change $(\Delta \mathrm{A})$ was smaller than $10^{-4}$ in the probe region of $450-1500 \mathrm{~nm}$.

\section{Results and discussion}

The results obtained in the native LH1 and the reconstituted LH1(Spx) show that the effects due to the reconstitution and other kinds of carotenoids are negligibly small. Therefore, it is concluded that the reconstituted spirilloxanthin in LH1(Spx) is identical to that in the native LH1. The reconstitution of purified carotenoids is a powerful technique to study the energy transfer and relaxation dynamics in the LH1 complex.

Absorbacne changes of spirilloxanthin after the Car $\mathrm{S}_{2}$ pump are similar in LH1 and solution as shown in Fig.2. Initially, the transient absorption due to $S_{2}$ appears in near-infrared region. $S_{2}$ relaxes to $S_{1}$ with a time constant of $80 \mathrm{fs}$. The transient absorption due to $S_{1}$ appears at $620 \mathrm{~nm}$ in LH1 and at $600 \mathrm{~nm}$ in solution. Difference of the absorption peak is due to influence of the surrounding proteins in LH1. The lifetime of $\mathrm{S}_{1}$ is $1.3 \mathrm{ps}$ in LH1 and $1.4 \mathrm{ps}$ in solution. The transient absorption at $5.0 \mathrm{ps}$ has a peak at shorter wavelength than the $\mathrm{S}_{1}$ absorption. It has been termed $\mathrm{S}^{*}$ both in LH1 and solution because of similaities of the spectra and decay time (about $5 \mathrm{ps}$ ), but in this study we used $\mathrm{S}_{\mathrm{LH} 1}$ and $\mathrm{S}_{\text {sol }}$, respectively. $\mathrm{S}_{\mathrm{LH} 1}$ is followed by the long-lived signal assigned to the triplet excited state $\left(\mathrm{T}_{1}\right)$, while spirilloxanthin in solution does not have the long-lived signal.
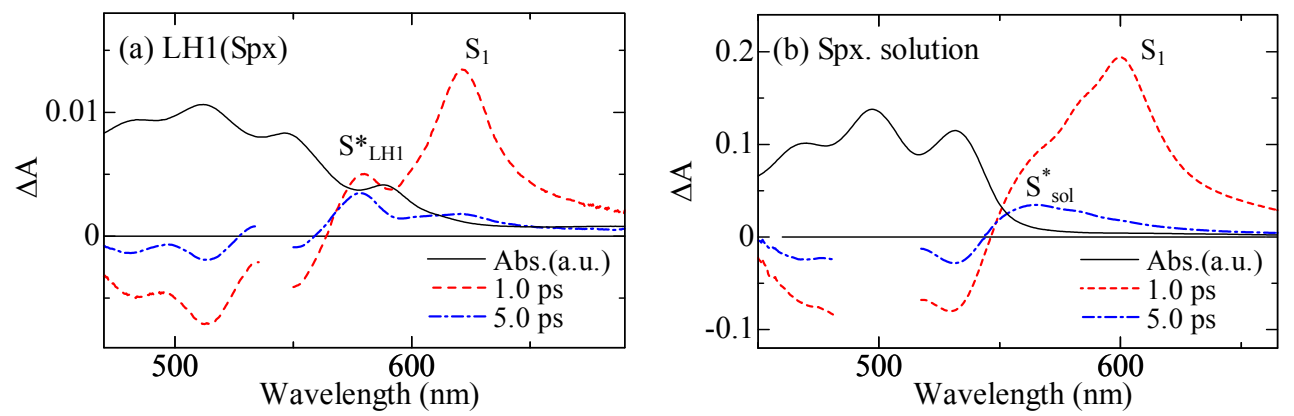

Fig. 2. Absorbance change of (a) LH1 and (b) spirilloxanthin solution after the Car $\mathrm{S}_{2}$ pump. Stationary absorption spectra are shown together (thin solid black curves).

FSRS signals on the Stokes side in LH1 are shown in Fig.3. Two intense signals without the Car $\mathrm{S}_{2}$ pump (Noex.) are assigned to $v_{1}\left(\mathrm{C}=\mathrm{C}\right.$ stretch.) and $v_{2}\left(\mathrm{C}-\mathrm{C}\right.$ stretch.) modes of $\mathrm{S}_{0}$. At $1.0 \mathrm{ps}$ after the Car $S_{2}$ pump, broad new signals are observed around 1770 and $1240 \mathrm{~cm}^{-1}$ by the $S_{1}$ resonant Raman pump (Fig.3(a)). Since their decay times are equal to the $S_{1}$ lifetime, they are assigned to the 
$v_{1}$ and $v_{2}$ modes of $S_{1}$, respectively. The high-frequency shift in $S_{1}$ is explained in terms of the vibronic coupling through the vibrational mode with $\mathrm{A}_{\mathrm{g}}$ symmetry. On the other hand, the signal obtained by the $\mathrm{S}_{\mathrm{LH} 1}$ resonant Raman pump (Fig.3(b)) does not have the $\mathrm{S}_{1}$ signal but has a peak at $1264 \mathrm{~cm}^{-1}$. The peak decreases slightly with a time constant of $5 \mathrm{ps}$ and shifts to $1272 \mathrm{~cm}^{-1}$. The $1264 \mathrm{~cm}^{-1}$ mode and the long-lived $1272 \mathrm{~cm}^{-1}$ mode are assigned to $\mathrm{S}_{\mathrm{LH} 1}$ and $\mathrm{T}_{1}$, respectively. $\mathrm{S}_{\mathrm{LH} 1}$ has similar vibrational modes to $\mathrm{T}_{1}$.
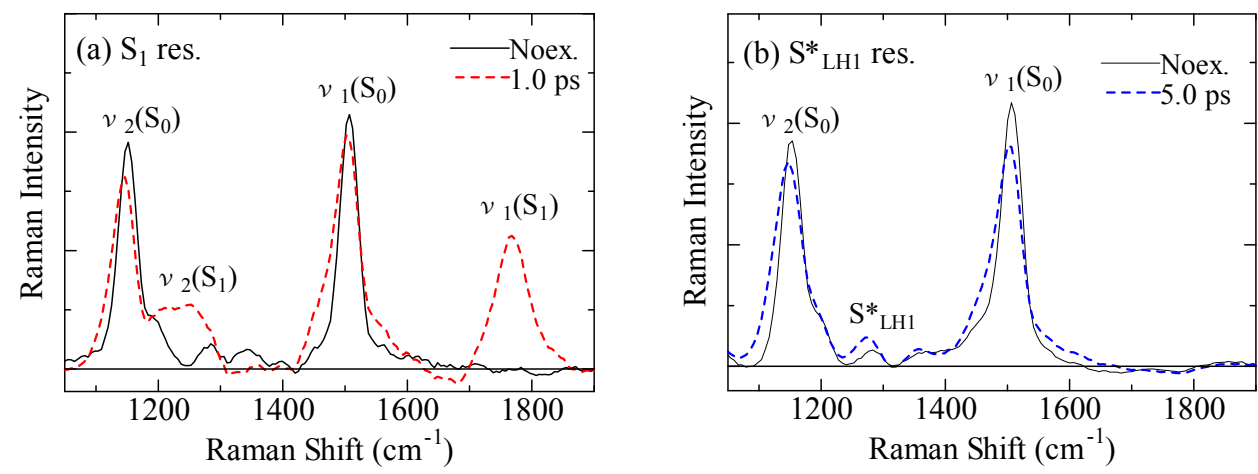

Fig. 3. FSRS signals of LH1 obtained by (a) the $620 \mathrm{~nm}$ Raman pump ( $\mathrm{S}_{1}$ resonance) and (b) the $580 \mathrm{~nm}$ Raman pump ( $\mathrm{S}_{\mathrm{LH} 1}$ resonance) after the Car $\mathrm{S}_{2}$ pump. Noex: without the Car $\mathrm{S}_{2}$ pump.

FSRS signals of spirilloxanthin in solution was measured using the $600 \mathrm{~nm}\left(\mathrm{~S}_{1}\right.$ resonance) and $560 \mathrm{~nm}\left(\mathrm{~S}^{*}\right.$ sol resonance) Raman pump pulses. The observed $\mathrm{S}_{1}$ Raman signal is almost identical to that in LH1. On the other hand, the signal induced by the $\mathrm{S}^{*}$ sol resonant Raman pump does not have the $1264 \mathrm{~cm}^{-1}$ and long-lived $1272 \mathrm{~cm}^{-1}$ modes. The Raman signal of $\mathrm{S}^{*}$ sol obtained by global fitting are almost equal to those of the $\mathrm{S}_{0}$ ground state except small low energy shift. It is concluded that $\mathrm{S}_{\text {sol }}^{*}$ is different from $\mathrm{S}_{\mathrm{LH} 1}$. It may be the hot $\mathrm{S}_{0}$ state.

\section{Conclusion}

Resonant FSRS has revelaed the vibrational dynaimcs in Car. The $\mathrm{S}_{1}$ state has almost same dynamics in LH1 and solutuon. The $S^{*}{ }_{\text {LH1 }}$ state has similar vibrational modes with the $T_{1}$ state. On the other hand, the $\mathrm{S}^{*}{ }_{\text {sol }}$ state is different from the $\mathrm{S}_{\mathrm{LH} 1}$ state and is suggested to be the vibrationaly excited ground state $\left(\right.$ hot $\left.\mathrm{S}_{0}\right)$.

\section{References}

1. R.J. Cogdell, A. Gall, and J. Köhler, Quart. Rev. Biophys. 39, 3, 2006.

2. T. Polívka and V. Sundström, Chem. Rev. 104, 2021, 2004.

3. E. Papagiannakis, J.T.M. Kennis, I.H.M. van Stokkum, R.J. Cogdell, and R. van Grondelle, Proc. Natl. Acad. Sci. USA 99, 6017, 2002.

4. H. Cong, D.M. Niedzwiedzki, G.N. Gibson, A.M. LaFountain, R.M. Kelsh, A.T. Gardiner, R.J. Cogdell, and H.A. Frank, J. Phys. Chem. B 112, 10689, 2008.

5. R. Nakamura, K. Nakagawa, M. Nango, H. Hashimoto, and M. Yoshizawa, J. Phys. Chem. B 115, 3233, 2011.

6. M. Yoshizawa and M. Kurosawa, Phys. Rev. A 61, 013808, 2000.

7. K. Nakagawa, S. Suzuki, R. Fujii, A.T. Gardiner, R.J. Cogdell, M. Nango, and H. Hashimoto, J. Phys. Chem. B 112, 9467, 2008. 